\title{
Decompressive C1 Laminectomy without Fusion for the Treatment of Craniovertebral Junction Stenosis with Myelopathy: Could It be One of Option?
}

\author{
Seon-Ah Choi ${ }^{1}$, Kyung-Hyun Kim¹, Un-Yong Choi ${ }^{2}$, Jeong-Yoon Park ${ }^{1}$, Sung-Uk Kuh', Dong-Kyu Chin ${ }^{1}$, \\ Keun-Su $\mathrm{Kim}^{1}$, Yong-Eun Cho \\ ${ }^{1}$ Department of Neurosurgery, Gangnam Severance Hospital, Spine and Spinal Cord Institute, Yonsei University College \\ of Medicine, Seoul; \\ ${ }^{2}$ Department of Neurosurgery, CHA Bundang Medical Center, CHA University School of Medicine, Seongnam, Republic \\ of Korea
}

Corresponding author:

Kyung-Hyun Kim

Department of Neurosurgery,

Gangnam Severance Hospital, Spine and Spinal Cord Institute, Yonsei

University College of Medicine, 211

Eonju-ro, Gangnam-gu, Seoul 06273,

Republic of Korea

Tel: +82-2-2019-3390

Fax: +82-3-3461-9229

E-mail: nskhk@yuhs.ac

Received: June 23, 2019

Revised: August 14, 2019

Accepted: August 20, 2019

\begin{abstract}
Objective: Trans-oral approach or occipitocervical/atlantoaxial fusion with/without posterior decompression has been considered to be an appropriate surgical strategy for craniovertebral junction (CVJ) stenosis with myelopathy. However, decompressive C1 laminectomy without posterior stabilization was reported recently for treating retro-odontoid pseudotumor. This study aimed to evaluate surgical outcomes of the patients treated with decompressive $\mathrm{C} 1$ laminectomy without posterior stabilization for CVJ stenosis with myelopathy. Methods: Ten patients underwent decompressive C1 laminectomy without posterior stabilization for CVJ stenosis with myelopathy from August 2007 to December 2016. All patients were evaluated preoperatively for spinal canal stenosis, cord signal changes, and instability based on preoperative computed tomography, magnetic resonance imaging, and plain dynamogram. We retrospectively reviewed the clinic charts and radiographs for investigating clinical outcomes such as the visual analog scale (VAS), and Ranawat grade scale and complications. Radiographic parameters including pre- and postoperative atlas-dens interval change in flexion and extension, O-C2 angle, C2-C7 Cobb angle, and C2-C7 sagittal vertical axis were measured. Results: The mean follow-up time was 41 months. Eight men and 2 women with a mean age of 58 years (range, 45-69 years) were enrolled. Preoperative neck pain by the VAS was improved significantly in all patients $(p<0.01)$. Nine of 10 patients showed improvement on the Ranawat grading scale, but 1 patient who required a Halo-vest due to aggravated instability after surgery remained unchanged. The statistical results of the preoperative and postoperative radiographic measurements were not significant. Conclusion: In select patients with certain indications, decompressive C1 laminectomy could be a viable option, especially in the elderly, patients with comorbidity, and patients with poor bone quality.
\end{abstract}

Key Words: Cervical vertebrae; Decompression, surgical; Laminectomy; Spinal stenosis

\section{INTRODUCTION}

Craniovertebral junction (CVJ) stenosis with myelopathy has a significant detrimental impact on patient quality of life, and can produce severe neck pain, quadriparesis, and respiratory dysfunction. Patients with untreated CVJ stenosis are less likely to survive long-term and may be bedridden ${ }^{18)}$. Patients with nonsurgical and conservative treatment had worse activities of daily living scores and were completely bedridden or deceased by the time of the final follow-up examination ${ }^{16)}$. Surgery contributes to prolonging the life span, improvement of pain and quality of life, and reversal of neurological deterioration ${ }^{15)}$.

CVJ stenosis has been considered a secondary spinal event related to primary instability at the atlantoaxial joint ${ }^{5,9,21)}$. Based on this proposed biomechanical mechanism of CVJ stenosis, the surgical methods considered are decompression or fusion. Therefore, the majority of surgeons have selected the trans-oral approach or occipitocervical/atlantoaxial fusion with or without posterior decompression, which has been considered an appropriate surgical strategy for CVJ stenosis ${ }^{1,2,4,11)}$. These surgical methods have a high mortality rate in early postoperative period of $4 \%$ to $17 \% \%^{14,15)}$. In addition, problems such as loosening of screws, complications due to prolonged operative time, limita- 
tion of neck movement, and adjacent segment degeneration can occur. This can be a major issue for the elderly, especially those with underlying comorbidities or poor bone quality.

Recently, some surgeons have reported decompressive C1 laminectomy without posterior stabilization ${ }^{10,19)}$. So far, the C1 laminectomy without posterior stabilization method has been performed only in retro-odontoid pseudo-tumor. We considered a simple decompressive laminectomy without posterior stabilization method in patients with CVJ stenosis with myelopathy, which is especially associated with instability, to provide longterm preserved stability. Thus this could be an additional option for surgical treatment in the elderly patient or patient with severe comorbidity. This study was designed to evaluate the clinical and radiographic outcomes of patients treated with decompressive $\mathrm{C} 1$ laminectomy without posterior stabilization for CVJ stenosis with myelopathy for over 3 years follow-up.

\section{MATERIALS AND METHODS}

\section{Patients}

This study was approved by our Institute Review Board. From August 2007 to December 2016, we performed decompressive C1 laminectomy without posterior stabilization for CVJ stenosis with myelopathy in 10 patients. All patients were evaluated preoperatively for spinal canal stenosis, cord signal change, and instability based on preoperative computed tomography, magnetic resonance imaging, and plain dynamogram. We retrospectively reviewed the clinic charts and radiographs for investigating clinical outcomes such as patient age, sex, neck and arm pain by visual analog scale (VAS), clinical symptoms, and complication. Preoperative and final follow-up neurological were graded on the Ranawat scale ${ }^{17)}$ (Tables 1, 2).

Radiographic parameters including pre- and postoperative atlas-dens interval (ADI) change in flexion and extension, occi-
pito-C2 angle (O-C2A) angle (O-C2A), C2-C7 Cobb angle, and $\mathrm{C} 2-\mathrm{C} 7$ sagittal vertical axis (SVA) were measured. The O-C2A, C2-C7 Cobb angle, and C2-7 SVA parameters have been documented elsewhere ${ }^{3,8,13)}$ (Fig. 1). Briefly, the O-C2A indicates the angle between McGregor's line and the inferior vertebral endplate line of $\mathrm{C} 2$. The $\mathrm{C} 2-\mathrm{C} 7 \mathrm{Cobb}$ angle indicates the angle between the inferior endplates of the $\mathrm{C} 2$ and $\mathrm{C} 7$ vertebral bodies. If the $\mathrm{C} 7$ vertebra was included in the fused vertebrae, we used the C2-end vertebrae of the fused segments below the C7 angle for analysis. In both measurements, a positive value indicates lordosis at the local segment.

The differences in ADI are defined as the differences between the values in flexion and value in extension. Regarding surgical factors, we evaluated operation time, blood loss, and intraoperative complications.

\section{Statistical Analysis}

The operation was performed with the patient in the prone position. The $\mathrm{C} 1$ laminectomy range was just before both lateral masses of the C1 lamina. In 3 of 10 patients, Partial additional C2 laminectomy was needed. If the fibrous adhesive band is

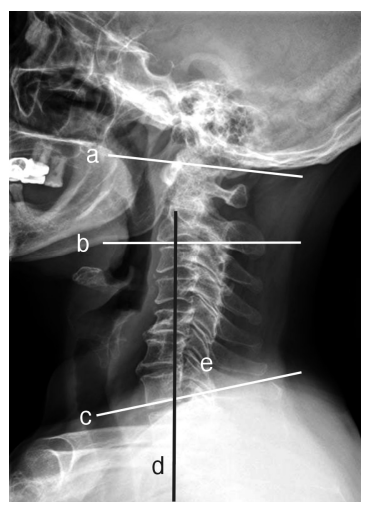

Fig. 1. Occipito-C2 anglewas defined as the angle between McGregor's line (a) and the inferior endplate line in the $\mathrm{C}$ (b), and C2-C7 Cobb angel measured between the interior endplate of C2 (b) and C7 (c). The C2-C7 sagittal vertical axis was defined as the deviation of the $\mathrm{C} 2$ plumb line $(\mathrm{d}$, extending from the centroid of the $\mathrm{C} 2$ vertebra) from the posterior superior end plate of $\mathrm{C} 7(\mathrm{e})$.

Table 1. Summary of patient demographics

\begin{tabular}{|c|c|c|c|c|c|}
\hline $\mathrm{No}$ & Age & Gender & Diagnosis & Surgery & "Follow-up (month) \\
\hline 1 & 63 & M & CSM & TL C1, STL C2 upper & 14 \\
\hline 2 & 61 & $\mathrm{~F}$ & Pseudotumor & $\mathrm{TL} \mathrm{Cl}$ & 22 \\
\hline 3 & 68 & M & Ankylosing spondylitis & $\mathrm{TL} \mathrm{Cl}$ & 17 \\
\hline 4 & 56 & $\mathrm{~F}$ & CSM & $\mathrm{TL} \mathrm{Cl}$ & 36 \\
\hline 5 & 56 & M & C1-2instability & $\mathrm{TL} \mathrm{Cl}$ & 29 \\
\hline 6 & 52 & M & Tb deformity & TL C1, STL C2 upper & 92 \\
\hline 7 & 45 & M & Traumatic deformity & $\mathrm{TL} \mathrm{Cl}$ & 102 \\
\hline 8 & 67 & M & Traumatic deformity & TL C1, STL C2 upper & 87 \\
\hline 9 & 69 & M & Klippel-Feil syndrome & $\mathrm{TL} \mathrm{C1,C2}$ & 10 \\
\hline 10 & 61 & M & Klippel-Feil syndrome & SOD, TL Cl & 8 \\
\hline
\end{tabular}

M: male; F: female; CSM: cervical spondylotic myelopathy; Tb: tuberculosis; TL: total laminectomy; STL: subtotal laminectomy; SOD: suboccipital decompression. 
still noted to be compressing the spinal cord intraoperatively, it should be removed after laminectomy. Following surgery, a Philadelphia neck collar was used for all patients for 3 months postoperatively. Additionally, patients were instructed to be careful about excessive flexion, extension, and impact.

\section{RESULTS}

\section{Clinical Outcomes}

The mean follow-up time was 41 months. Eight men and 2 women with a mean age of 58 years (range, 45-69 years) were included in this study. Pre- and postoperative VAS was used to evaluate for neck pain and significantly improved from a mean 5.8 to a mean 1.8 after surgery $(\mathrm{p}<0.01)$. Nine of 10 patients showed improvement on the Ranawat grading scale, but 1 patient remained unchanged (Ranawat grade IIIb). The patient showed a preoperative ADI in flexion of $9.55 \mathrm{~mm}$, extension of $2.7 \mathrm{~mm}$, and a 6-month postoperative ADI of $11.56 \mathrm{~mm}$ and $11.17 \mathrm{~mm}$, respectively. Eventually, the patient required a Halo-vest due to aggravated instability (Table 3).

\section{Radiologic Outcome}

Atlantoaxial instability was defined as an ADI $>4 \mathrm{~mm}$, according to the criteria described by White and Panjabi. The number of patients with an $\mathrm{ADI}$ in flexion $>4 \mathrm{~mm}$ was 3 before surgery and 4 after surgery in this study. The number of patients showing a difference between flexion and extension in ADI of $>3 \mathrm{~mm}$ was 3 before surgery and 2 after surgery. The average OC2 angle was $20.78^{\circ}$ preoperatively and $19.23^{\circ}$ postoperatively, and the preoperative and postoperative difference was $1.55^{\circ}$. The mean C2-C7 Cobb angle was $22.83^{\circ}$ preoperatively and $24.39^{\circ}$ postoperatively, and the preoperative and postoperative difference was $-1.56^{\circ}$. The preoperative $\mathrm{C} 2-\mathrm{C} 7 \mathrm{SVA}$ mean was $16.09 \mathrm{~mm}$ and postoperative mean was $17.25 \mathrm{~mm}$. The statistical results of the preoperative and postoperative radiographic measurements (ADI, OC2 angle, C2-C7 Cobb angle, and $\mathrm{C} 2-\mathrm{C} 7$ SVA) showed no significant differences. The mean surgical time was $117.3 \pm 22.7 \mathrm{~min}$, and the mean blood loss was $102 \pm$ $44.6 \mathrm{~mL}$. There was no documentation of surgical complication such as dura tear, vertebral artery-related injury, and intraope-

Table 2. Ranawat scale of the patient impairment with spinal cord compression

\begin{tabular}{cl}
\hline \hline Grade & \multicolumn{1}{c}{ Clinical finding } \\
\hline 1 & No neural deficit \\
2 & Subjective weakness with hyper-reflexia and dysesthesia \\
3 & Objective finding of weakness and long tract signs \\
$3 A$ & Could walk \\
$3 B$ & Not ambulatory \\
\hline
\end{tabular}

rative cord injury in any patient during the postoperative course (Fig. 2, Table 4).

\section{DISCUSSION}

The CVJ is the most mobile area of the human body. The
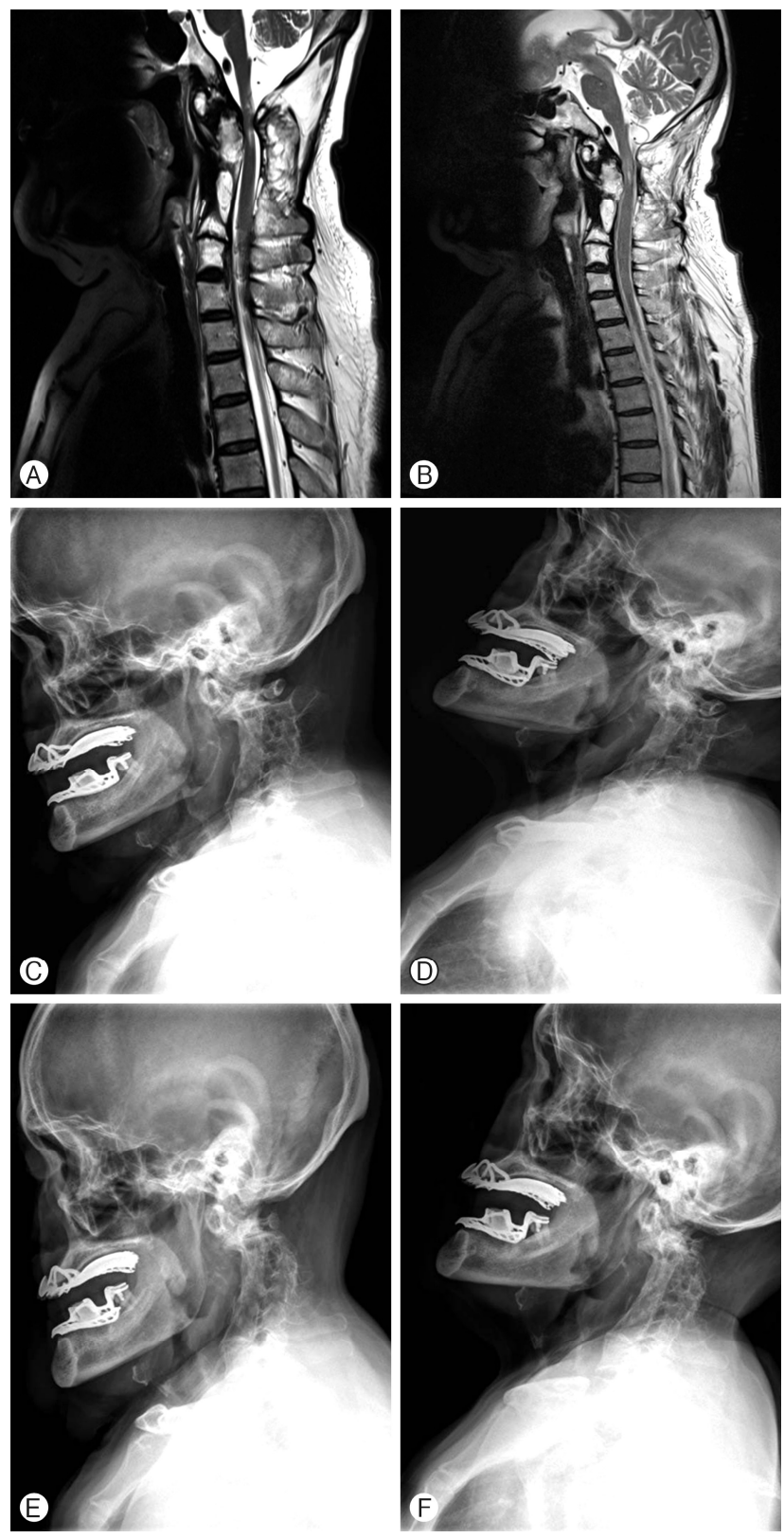

Fig. 2. A 68 year-old man. Cenvical stenosis with cord signal change was revealed by sagittal image on T2-weighted magnetic resonance imaging (A). Follow $\mathrm{Cl}$ laminectomy (B), cord compression is released (D). Preoperative (C, D) and postoperative $(E, F)$ lateral dynamic radiographs that were obtained in the flexion $(C, E)$ and extension $(D, F)$. Comparing preoperative and postoperative dynamic radiographs, $(C, E)$ vs. (D, F), additional atlantoaxial instability is not seen. 
CVJ is composed of 3 components: bone (occipital bone, atlas, and axis), joint, and circumjacent ligaments. The relationship between these components is complex and difficult to understand. The CVJ protects the cervical spinal cord, brain stem, and lower cranial nerves, and concurrently provides excellent flexibility in the O-C1 and C1-2 joints. Specifically, the O-C1 joint represents $50 \%$ of the total flexion-extension motion in the entire cervical spine, while the C1-2 joint accounts for $50 \%$ of the rotational motion ${ }^{10,20)}$. The ligaments are the main contributor to mechanical stability of the CVJ. The transverse and alar ligaments attaching to the posterior odontoid process are especially important to control the CVJ. The posterior occipito-atlantal membrane and posterior atlantoaxial membrane are attached to the posterior arch of the atlas; however, these membranes are less important than the transverse and alar ligaments for stabilizing the CVJ biomechanics. The degeneration of the articular cartilage, reduction in joint space, and secondary incompetence of ligaments controlling movements probably lead to atlantoaxial instability ${ }^{4,7,12)}$. Atlantoaxial joint arthritis is likely initiated or exacerbated by a subtle trauma. The clinical features suggest that the process of joint degeneration and instability are progressive phenomena and extend over several months to years.

Regarding these pathobiomechanical causes and concerns about increased instability, CVJ stenosis has been treated with the posterior stabilization method. The posterior stabilization method for CVJ stenosis is the most common treatment, but it has distinct disadvantages, such as the need for instrumentation, morbidity due to bone grafting, a restricted range of neck movement, dyspnea, and dysphagia ${ }^{6,13,22)}$. As observed in this study, C1 decompressive laminectomy without posterior stabilization shows satisfactory clinical, neurological, and surgical outcomes without severe complication.

While CVJ stenosis is often associated with ossification of anterior longitudinal ligament, ossification of posterior longitudinal ligament, and severe cervical spondylosis, the cervical range of motion (ROM) of patients with CVJ stenosis is fundamentally disturbed. A posterior fusion would lead to further reduction in ROM, and thus should be avoided. Based on these anatomical properties and the results of this study, C1 laminectomy without posterior stabilization method is not considered to induce postoperative atlantoaxial instability. Rather, it produced rapid recovery in its short operation time and reduced operative bleeding, and patients showed neurological and clinical improvement.

On the other hand, it is crucial to maintain stability in C1 laminectomy without posterior stabilization because long-term degenerative changes such as facet hypertrophy or fusion that result in restabilization of existing structures may occur. In the same manner, several radiological indexes implying instabi-

Table 3. Result of surgical treatment

\begin{tabular}{|c|c|c|c|c|c|c|}
\hline & \multicolumn{2}{|c|}{ Visual analog scale } & \multicolumn{2}{|c|}{ Ranawat scale } & \multicolumn{2}{|c|}{ Complication } \\
\hline & Preoperative & Last follow-up & Preoperative & Last follow-up & Intraoperative & Postoperative \\
\hline 1 & 5 & 0 & $3 A$ & 2 & - & - \\
\hline 2 & 8 & 3 & $3 \mathrm{~A}$ & 2 & - & - \\
\hline 3 & 6 & 2 & $3 \mathrm{~A}$ & 2 & - & - \\
\hline 4 & 7 & 3 & $3 \mathrm{~A}$ & 2 & - & - \\
\hline 5 & 5 & 2 & $3 B$ & $3 B$ & - & Halo-vest \\
\hline 6 & 4 & 1 & 2 & 1 & - & - \\
\hline 7 & 5 & 2 & $3 \mathrm{~A}$ & 1 & - & - \\
\hline 8 & 3 & 0 & $3 \mathrm{~A}$ & 2 & - & - \\
\hline 9 & 8 & 2 & $3 A$ & 2 & - & - \\
\hline 10 & 7 & 3 & $3 \mathrm{~A}$ & 2 & - & - \\
\hline
\end{tabular}

Table 4. Radiologic and intraoperative outcomes

\begin{tabular}{lccc}
\hline \hline & Preoperation & Postoperation & p-value \\
\hline ADI $(\mathrm{mm})$ & $3.98 \pm 2.70$ & $4.04 \pm 3.24$ & 0.805 \\
O-C2 angle & $20.78 \pm 14.15$ & $19.23 \pm 16.55$ & 0.631 \\
C2-C7 Cobb angle & $22.83 \pm 15.85$ & $24.39 \pm 13.81$ & 0.686 \\
C2-C7 SVA (mm) & $16.09 \pm 21.77$ & $17.25 \pm 21.30$ & 0.414 \\
Mean operation time (min) & & $117.3 \pm 22.7$ & \\
Mean blood loss $(\mathrm{mL})$ & & $102.0 \pm 44.6$ & \\
\hline
\end{tabular}

ADI: atlas-dens interval; SVA: sagittal vertical axis. 
lity-basion-axial interval less than $12 \mathrm{~mm}$, basion-dens interval less than $9 \mathrm{~mm}$, atlanto-occipital interval less than $4 \mathrm{~mm}$, ADI over $3 \mathrm{~mm}$, flexion/extension ADI difference over $9 \mathrm{~mm}$ - are generally considered more suitable for occipitocervical fusion.

Therefore, although the exact indication is not yet defined, we could expect that patients with old age, combined comorbidity, poor bone quality, posterior compression as their main problem and previous chronic degenerative changes enough to prevent instability could be good candidates for C1 decompressive laminectomy without posterior stabilization. However, longer-term follow-up is needed to assess the potential instability of the CVJ. One patient needed additional treatment after surgery, but this patient may not have had the proper indications. Considerable instability existed preoperatively but was neglected in case of revisional additional fusion after C1 laminectomy. The revision surgery was not difficult as only fixation and fusion is needed.

Recently, 2 publications have reported patients with retroodontoid mass lesions that were treated with C1 laminectomy without posterior stabilization ${ }^{10,19)}$. They also showed good clinical and neurological outcomes without resultant atlantoaxial instability. Considering these study results, C1 decompressive laminectomy without posterior stabilization may be an effective and safe surgical alternative. In our study, the final follow up results showed good clinical and neurological outcomes in all patients except for one, in whom a Halo-vest was required. Regarding atlantoaxial instability, instability progression was observed in 1 of 10 patients, although this did not result in any clinical issues and neurological outcome improved. These good clinical and neurological outcomes show the suitability of this surgical option in treating this pathology.

There are some limitations of this study. Selection bias and a small group are the primary limitations, as the study was a retrospective chart review of 10 patients. In the future, an analysis of risk factors for aggravation of postoperative instability should incorporate a large number of patients. Providing biomechanical evidence for $\mathrm{C} 1$ laminectomy without pos- terior fusion and its effect on instability is necessary. In addition, the appropriate indications for the surgical plan are crucial, and thus prospective randomized studies will be needed in the future.

\section{CONCLUSION}

Surgical intervention remains the most effective therapeutic option for patients with CVJ stenosis with myelopathy. In this study, patients showed improvement in clinical and neurological outcomes, even when presenting preoperatively with atlanto-axial instability. In select patients with certain indications, decompressive C1 laminectomy without posterior stabilization could be viable option, especially in elderly patients, and patients with comorbidity and/or poor bone quality.

\section{CONFLICTS OF INTEREST}

No potential conflict of interest relevant to this article was reported.

\section{REFERENCES}

1. Birch BD, Khandji AG, McCormick PC: Atlantoaxial degenerative articular cysts. J Neurosurg 85:810-816, 1996

2. Cai CY, Palmer CA, Paramore CG: Exuberant transverse ligament degeneration causing high cervical myelopathy. J Spinal Disord 14:84-88, 2001

3. Carreon LY, Smith CL, Dimar JR, 2nd, Glassman SD: Correlation of cervical sagittal alignment parameters on full-length spine radiographs compared with dedicated cervical radiographs. Scoliosis Spinal Disord 11:12, 2016

4. Finn M, Fassett DR, Apfelbaum RI: Surgical treatment of nonrheumatoid atlantoaxial degenerative arthritis producing pain and myelopathy. Spine (Phila Pa 1976) 32:3067-3073, 2007

5. Goel A: Craniovertebral junction instability: A review of facts about facets. Asian Spine J 9:636-644, 2015

6. Huang M, Gonda DD, Briceno V, Lam SK, Luerssen TG, Jea A: Dyspnea and dysphagia from upper airway obstruction after occipitocervical fusion in the pediatric age group. Neurosurg Focus 38:E13, 2015

7. Isono M, Ishii K, Kamida T, Fujiki M, Goda M, Kobayashi H: Retro-odontoid soft tissue mass associated with atlantoaxial subluxation in an elderly patient: a case report. Surg Neurol 55:223227, 2001

8. Izeki M, Neo M, Takemoto M, Fujibayashi S, Ito H, Nagai K, et al.: The O-C2 angle established at occipito-cervical fusion dictates the patient's destiny in terms of postoperative dyspnea and/or dysphagia. Eur Spine J 23:328-336, 2014

9. Jun BY, Yoon KJ, Crockard A: Retro-odontoid pseudotumor in diffuse idiopathic skeletal hyperostosis. Spine (Phila Pa 1976) 27:E266-E270, 2002

10. Kakutani K, Doita M, Yoshikawa M, Okamoto K, Maeno K, Yurube T, et al.: C1 laminectomy for retro-odontoid pseudotumor without atlantoaxial subluxation: review of seven consecutive cases. Eur Spine J 22:1119-1126, 2013

11. Kaufmann AM, Halliday WC, West M, Fewer D, Ross I: Periodontoid synovial cyst causing cervico-medullary compression. Can J Neurol Sci 23:227-230, 1996

12. Lagares A, Arrese I, Pascual B, Gòmez PA, Ramos A, Lobato $\mathrm{RD}$ : Pannus resolution after occipitocervical fusion in a nonrheumatoid atlanto-axial instability. Eur Spine J 15:366-369, 2006

13. Miyata M, Neo M, Fujibayashi S, Ito H, Takemoto M, Nakamura T: O-C2 angle as a predictor of dyspnea and/or dysphagia after occipitocervical fusion. Spine (Phila Pa 1976) 34:184-188, 2009

14. Mizutani J, Tsubouchi S, Fukuoka M, Otsuka T, Matsui N: Surgical treatment of the rheumatoid cervical spine in patients aged 70 years or older. Rheumatology (Oxford) 41:910-916, 2002

15. Nannapaneni R, Behari S, Todd NV: Surgical outcome in rheumatoid Ranawat Class IIIb myelopathy. Neurosurgery 56:706- 
715,2005

16. Omura K, Hukuda S, Katsuura A, Saruhashi Y, Imanaka T, Imai $\mathrm{S}$ : Evaluation of posterior long fusion versus conservative treatment for the progressive rheumatoid cervical spine. Spine (Phila Pa 1976) 27:1336-1345, 2002

17. Ranawat CS, OLeary P, Pellicci P, Tsairis P, Marchisello P, Dorr L: Cervical spine fusion in rheumatoid arthritis. J Bone Joint Surg Am 61:1003-1010, 1979

18. Sunahara N, Matsunaga S, Mori T, Ijiri K, Sakou T: Clinical course of conservatively managed rheumatoid arthritis patients with myelopathy. Spine (Phila Pa 1976) 22:2603-2607, 1997

19. Takemoto M, Neo M, Fujibayashi S, Sakamoto T, Ota M, Otsuki
B, et al.: Clinical and radiographic outcomes of C1 laminectomy without fusion in patients with cervical myelopathy that is associated with a retro-odontoid pseudotumor. Clin Spine Surg 29: E514-E521, 2016

20. Werne S: Studies in spontaneous atlas dislocation. Acta Orthop Scand Suppl 23:1-150, 1957

21. Yoshida K, Hanyu T, Takahashi HE: Progression of rheumatoid arthritis of the cervical spine: radiographic and clinical evaluation. J Orthop Sci 4:399-406, 1999

22. Yoshida M, Neo M, Fujibayashi S, Nakamura T: Upper-airway obstruction after short posterior occipitocervical fusion in a flexed position. Spine (Phila Pa 1976) 32:E267-E270, 2007 